\title{
EVALUACIÓN DEL COMPORTAMIENTO HIDRÁULICO DE DOCE MODELOS DE GOTEROS DE BAJO CAUDAL
}

\author{
EVALUATION OF BEHAVIOR OF TWELVE MODELS OF DROPPERS OF LOW \\ HYDRAULIC FLOW
}

\author{
${ }^{1}$ Rocío Pastor J. y ${ }^{2}$ Ángel Narro D.
}

\begin{abstract}
Resumen
La investigación se realizó en el laboratorio de Riego y Drenaje de la Facultad de Ingeniería Agrícola, de la Universidad Nacional Agraria La Molina. El objetivo fue determinar el coeficiente de variación y las curvas caudal-presión de 12 modelos de goteros. Las mangueras fueron evaluadas en un banco de pruebas que consta de una estructura de soporte que sostiene a la bandeja de captación. Las dimensiones son 2,0 $\mathrm{m}$ de largo por 1,0 $\mathrm{m}$ de ancho y la estructura de conducción de $0,45 \mathrm{~m}$ de largo por 1,0 $\mathrm{m}$ de ancho. Presenta 6 salidas con tees roscadas hembras de PVC de 3/4 de pulgada para tubos de $16 \mathrm{~mm}$. La evaluación de las líneas emisoras se realizó para 8 goteros autocompensados y 4 no compensados. Para la obtención del coeficiente de variación se utilizó una muestra de 30 goteros sometidos a un rango intermedio de regulación de presión para los autocompensados y de 1,0 bar de presión nominal para los otros. Las curvas características se determinaron a partir de una muestra de 4 goteros sometidos desde presión cero hasta 1,2 de la presión máxima, con tres repeticiones y a una temperatura del agua de $23 \pm 3^{\circ} \mathrm{C}$. Todas las evaluaciones se realizaron según la segunda edición de la norma ISO 9261 del año 2004, la cual brinda los requerimientos funcionales y mecánicos para emisores y tuberías emisoras. Comparando los resultados, solamente el modelo de manguera Tifdrip, con un caudal de 2,0 1/h, presentó un coeficiente de variación de $12,49 \%$, mientras que los otros modelos presentaron valores menores al 7\%. De acuerdo a los catálogos de los fabricantes, los modelos Taldrip 1.0, Taldrip 1.7, NewGR 2.0 y Corona 4.0 presentaron similares curvas caudal-presión, pero no el modelo Tifdrip 2.0.
\end{abstract}

Palabras claves: coeficiente de variación, curvas caudal-presión, emisores, líneas emisoras.

\begin{abstract}
The present research was performed in the Irrigation and Drainage Laboratory of Agricultural Engineering Faculty of Agraria La Molina University. The objective was to determinate the coefficient of variation and flow-pressure curves of 12 models of drippers that were undergoing evaluation. The driplines were evaluated in a testing bench, a support structure that holds the catchment tray. The dimensions are 2,0 by 1,0 meters and the conduction structure 0,45 by 1,0 meters. It has 6 outputs with PVC female threaded tees of $3 / 4$ inch to tubes of $16 \mathrm{~mm}$. The evaluation of 12 types of drippers, 8 pressure compensating drippers and 4 uncompensating. To obtain the coefficient of variation were used 30 drippers under intermediate range of pression regulation for the self-compensating and 1,0 bar for the uncompensating. The flow-pressure curves were determinate for a sample of 4 drippers from zero pressure to 1,2 of maximum pressure. All measurements were performed with three replicates and a water temperature of $23 \pm 3^{\circ} \mathrm{C}$. All evaluations were made according the second edition of the standard ISO 9261 (2004), which provides the functional and mechanical requirements for drippers. Comparing the results, only the model of tube Tifdrip of $2,0 \mathrm{l} / \mathrm{h}$, presented a higher coefficient of variation of $12,49 \%$, while the other dripper models presented values lowers than $7 \%$. According manufacturers catalogs, about characteristic curves of these drippers, the next models presented similar values of flow rate: Taldrip 1.0, Taldrip 1.7, NewGR 2.0 and Corona 4.0, but not the Tifdrip 2.0 model.
\end{abstract}

Key words: coefficient of variation, flow-pressure curves, drippers, driplines.

\section{Introducción}

Debido al aumento de la superficie cultivada, impulsada por el boom agro exportador principalmente a lo largo y ancho de la zona costera de nuestro país y los problemas de escasez del recurso hídrico por su ubicación en una zona desértica y por consiguiente de escasas precipitaciones y fuentes de agua, se hace de suma importancia el empleo de un sistema que permita el ahorro del agua a los cultivos. El riego presurizado por goteo presenta un notable ahorro del recurso hídrico, asegurando una aplicación directa a la zona radicular de los cultivos.

El método de riego por goteo se caracteriza por la aplicación de láminas de agua dirigidas al sistema radical, humedeciendo una zona restringida, formando un bulbo húmedo establecido por un gotero de baja descarga, operando en un determinado rango de presiones. La uniformidad y distribución del agua en este sistema de riego es importante porque influye en la eficiencia del uso 
de energía y aplicación de fertilizantes para alcanzar el máximo rendimiento del cultivo y aumentar la eficiencia del sistema de riego.

Los emisores son las estructuras hidráulicas a través de las cuales el agua sale del interior de los sistemas de riego presurizado hacia el ambiente, ya sea directamente al suelo o al aire. Debido a la gran variedad de modelos de emisores y de líneas emisoras que se comercializan en nuestro país y a que algunas casas distribuidoras de sistemas de riego proporcionan la información técnica de los emisores de manera incompleta, proporcionando en algunos casos las curvas caudal-presión y/o en menor medida el valor exacto del coeficiente de variación, es de suma relevancia la determinación del comportamiento hidráulico, directamente relacionado con la calidad de fabricación de estos elementos, determinados bajo condiciones de laboratorio, puesto que por medio de estas características se establece la idoneidad de emplear estos goteros para satisfacer la uniformidad y eficiencia de aplicación entre un gotero u otro que satisfaga la demanda de agua en las descargas y el comportamiento a diferentes presiones de los emisores al momento de entrar en funcionamiento para lograr un aumento de la productividad.

La información que se obtiene al finalizar las evaluaciones permite conocer las características de cada gotero, dato que debe tomarse en cuenta al momento de diseñar un sistema de riego, para alcanzar una entrega del agua de manera uniforme y con un volumen adecuado para satisfacer los requerimientos de riego de los cultivos. Los emisores son dispositivos que controlan la salida del agua desde las tuberías laterales. Su función es la de causar una caída de presión de tal manera que sólo un pequeño flujo de agua sea descargada.

Fuentes Yagüe, J. L. (2003) sostiene que "esta necesaria pérdida de carga es realizada a través del uso de orificios, vórtices, pasos tortuosos, placas de impacto o una combinación de esas". Según el caudal que proporcionan se dividen en dos grupos: emisores de bajo caudal (inferior a $16 \mathrm{l} / \mathrm{h}$ ) que comprende los goteros y las tuberías emisoras o cintas y los emisores de alto caudal (entre 16 y 200 1/h), que comprende los difusores y los microaspersores.

El objetivo de la investigación fue evaluar el comportamiento hidráulico de doce modelos de goteros de bajo caudal, determinando el coeficiente de variación de fabricación y sus curvas caudal-presión.

\section{Materiales y métodos}

La investigación se realizó en el laboratorio de Riego y Drenaje de la Facultad de Ingeniería Agrícola, de la Universidad Nacional Agraria La Molina. Las mangueras fueron evaluadas en un banco de pruebas que consta de una estructura de soporte que sostiene a la bandeja de captación. Las dimensiones son de 2,0 $\mathrm{m}$ de largo por $1,0 \mathrm{~m}$ de ancho y la estructura de conducción de $0,45 \mathrm{~m}$ de largo por 1,0 $\mathrm{m}$ de ancho. Presenta 6 salidas con tees roscadas hembras de PVC de $3 / 4$ de pulgada para tubos de $16 \mathrm{~mm}$.

La evaluación de las líneas emisoras se realizó para 8 goteros autocompensados: manguera Amnondrip (qn = $1,1 \mathrm{l} / \mathrm{h}$ ), manguera Amnondrip ( $\mathrm{qn}=1,6 \mathrm{l} / \mathrm{h}$ ), manguera Naan $(\mathrm{qn}=1,6 \mathrm{l} / \mathrm{h})$, manguera Naan $(\mathrm{qn}=2,2 \mathrm{l} / \mathrm{h})$, gotero Clicktif ( $q n=21 / h)$, gotero Clicktif $(q n=81 / h)$, manguera Topdrip ( $q n=1,0 \mathrm{l} / \mathrm{h}$ ), gotero Corona ( $\mathrm{qn}=4$ 1/h) y 4 no compensados: manguera Tifdrip ( $q n=21 / h)$, cinta Taldrip $(\mathrm{qn}=1,0 \mathrm{l} / \mathrm{h})$, cinta Taldrip $(\mathrm{qn}=1,7 \mathrm{l} / \mathrm{h})$, manguera New GR (qn $=2$ 1/h).

Todas las evaluaciones se realizaron según la segunda edición de la norma ISO 9261 del año 2004, la cual brinda los requerimientos funcionales y mecánicos para emisores y tuberías emisoras.

La determinación de la uniformidad del gasto de emisión (pruebas de coeficiente de variación) y de las curvas caudal-presión se hicieron con los 12 modelos de emisores, de los cuales 11 modelos (insertados y los integrados a las mangueras y cintas) fueron evaluados con espaciamiento de $40 \mathrm{~cm}$ entre emisor y emisor y solo el modelo de manguera NewGR contó con gotero integrado cada $50 \mathrm{~cm}$.

\section{Coeficiente de variación}

Según Medina San Juan, J. A. (1997), “todo proceso industrial es imperfecto, por tanto el producto elaborado experimenta variaciones, aunque siempre comprendida entre márgenes que se consideren tolerables". Pizarro, F. (1996), refiriéndose a la uniformidad en los emisores, indica que en teoría todos los emisores de una misma marca y modelo deberían dar el mismo caudal cuando actúan a la misma presión y temperatura, pero en la práctica no ocurre así. Las variables de fabricación (tipo de material, temperatura, desgaste de la maquinaria, etc.) afectan las dimensiones del emisor y su caudal.

Para la obtención del coeficiente de variación se utilizó una muestra de 30 goteros sometidos a un rango intermedio de presión para los autocompensados y de 1,0 bar de presión nominal para los no compensados.

Para determinar la presión de entrada de los emisores autocompensados se fijaron las presiones mínima y máxima de trabajo, manteniéndose ambas durante 3 min, repitiendo el proceso tres veces. Se determinó la presión correspondiente al valor medio del intervalo de regulación, la cual se mantuvo durante una hora, luego se determinaron los caudales de cada uno de los emisores.

Los volúmenes de agua se recepcionaron en envases de $500 \mathrm{ml}$ de capacidad, previa estabilización de la presión y durante un tiempo de diez, cinco (Corona 4,0) o cuatro minutos (Clicktiff 8,0), dependiendo de los caudales de cada gotero. Los envases fueron colocados al mismo tiempo y retirados de la misma manera. El volumen descargado se midió por medio de probetas graduadas.

Keller y Karmeli en 1974 introdujeron un coeficiente 
de variación de fabricación $(C V)$, como medida de la dispersión estadística en el proceso de fabricación de los emisores. Este $C V$ es un valor adimensional estimado, que se define como el cociente entre la desviación típica y el valor medio: $C V=\sigma / q a$, donde $\sigma$ la desviación típica de los caudales de la muestra $(1 / \mathrm{h})$ y $q a$ el caudal medio de la muestra $(1 / h)$, que no debe desviarse del caudal nominal ( $q n$ ) en más de un $\pm 7 \%$. El $C V$ no debe exceder del $7 \%$. Siendo estos nuevos valores los considerados en esta segunda edición de la norma ISO 9261.

\section{Curvas caudal-presión}

Fuentes Yagüe, J. L. (2003) indica que "el agua atraviesa el emisor a través de uno o varios conductos, cuya configuración determina su comportamiento hidráulico". En cualquier emisor el caudal de descarga y la presión de servicio se relacionan mediante la ecuación: $q=k h^{x}$, donde $q$ es el caudal del emisor $(1 / \mathrm{h}), k$ el coeficiente característico de cada emisor, que equivale al caudal que proporcionaría a una presión de $10 \mathrm{mca}, h$ la presión a la entrada del emisor (mca o Kpa) y $x$ el exponente de descarga propio de cada emisor, caracterizado por el régimen de flujo del emisor y/o de sus dispositivos de auto compensación. De acuerdo a Pizarro, F. (1996), los valores de $k$ y $x$ son característicos de cada tipo de emisor. Con frecuencia los fabricantes sólo informan acerca de un punto de la ecuación del emisor, pero debe exigírseles que proporcionen la ecuación con los valores numéricos de $k$ y $x$, así como el entorno de trabajo o por lo menos la curva caudal-presión.

En la Tabla 1 se muestran valores para $x$, según el emisor. Las curvas características se determinaron a partir de una muestra de 4 goteros sometidos desde presión cero hasta 1,2 de la presión máxima, con tres repeticiones y a una temperatura del agua de $23 \pm 3^{\circ} \mathrm{C}$. Los caudales obtenidos en la prueba del coeficiente de variación se ordenaron en sentido ascendente, seleccionándose los que ocuparon los lugares $2^{\circ}, 15^{\circ}, 16^{\circ}$ y $29^{\circ}$, es decir, descargas de inicio, final y dos valores medios. Luego se evaluó cada emisor, con incrementos de presión que no superaron en cada caso $50 \mathrm{kPa}$ y a presiones descendentes solo para los emisores compensados.

Tabla 1. Valores de x, según el emisor.

\begin{tabular}{lc}
\hline \multicolumn{1}{c}{ Emisor } & $\mathrm{x}$ \\
\hline Régimen laminar & 1 \\
Microtubos & $0,75-1$ \\
Helicoidal & $0,65-0,75$ \\
Régimen turbulento (orificio, de laberinto) & 0,5 \\
Vortex & 0,4 \\
Autocompensante & $0-0,4$ \\
Teórico perfectamente autocompensante & 0 \\
\hline
\end{tabular}

Se determinó el caudal medio $(q)$, para cada nivel de presión a la entrada $(p)$, obtenido a través de los caudales de los emisores a distintas presiones. El caudal medio para cada presión fue el promedio de las descargas de los 4 goteros previamente seleccionados; para el caso de goteros autocompensados fue la media de 8 descargas, es decir, a presiones tomadas tanto de manera ascendente como descendente.

Los caudales se aforaron, previa estabilización de la presión y en un tiempo de tres minutos. Los envases fueron colocados y retirados al mismo tiempo.

\section{Intervalos de confianza}

Gabriel Rada, citado por Merino, T. (2007), indica que en el contexto de estimar un parámetro poblacional, un intervalo de confianza es un rango de valores (calculado en una muestra) en el cual se encuentra el verdadero valor del parámetro, con una probabilidad determinada.

La probabilidad que el verdadero valor del parámetro se encuentre en el intervalo construido se denomina nivel de confianza y se denota con $1-\alpha$. La probabilidad de equivocarse se llama nivel de significancia y se simboliza con $\alpha$. Generalmente se construyen intervalos con confianza $1-\alpha=95 \%$ (o significancia $\alpha=5 \%$ ). Menos frecuentes son los intervalos con $\alpha=10 \%$ o $\alpha=1 \%$.

Para construir un intervalo de confianza, se puede comprobar que la distribución Normal Estándar cumple $P(-1,96<z<1,96)=0,95$. Luego, si una variable $X$ tiene distribución $\mathrm{N}\left(\mu, \sigma^{2}\right)$, entonces el $95 \%$ de las veces se cumple:

$$
-1,96 \leq \frac{X-\mu}{\sigma} \leq 1,96
$$

El resultado es un intervalo que incluye al $\mu$ el $95 \%$ de las veces. Es decir, es un intervalo de confianza al 95\% para la media $\mu$ cuando la variable $X$ es normal y $\sigma^{2}$ es conocido. Generalmente, cuando se quiere construir un intervalo de confianza para la media poblacional $\mu$, la varianza poblacional $\sigma^{2}$ es desconocida, por lo que el intervalo para $\mu$ construido al final es muy poco práctico. Si en el intervalo se reemplaza la desviación estándar poblacional $\sigma$ por la desviación estándar muestral s, el intervalo de confianza toma la forma:

$$
X-1,96 * \frac{s}{\sqrt{n}} \leq \mu \leq X+1,96 * \frac{s}{\sqrt{n}}
$$

La cual es una buena aproximación para el intervalo de confianza de $95 \%$ para $\mu$ con $\sigma^{2}$ desconocido. Esta aproximación será mejor cuando el tamaño de la muestra sea grande.

Cuando el tamaño de muestra es pequeño $(\mathrm{n}<30)$, el intervalo de confianza requiere utilizar la distribución t de Student (con $n-1$ grados de libertad, siendo $n$ el tamaño de la muestra), en vez de la distribución normal (por ejemplo, para un intervalo de $95 \%$ de confianza, los límites del intervalo ya no serán construidos usando el 
valor 1,96$)$

\section{Resultados y discusión \\ Coeficiente de variación}

Se determinaron los coeficiente de variación de fabricación para cada uno los emisores, con el fin de realizar la comparación de acuerdo a la segunda edición de la norma ISO 9261 (2004). En la Tabla 2 se muestran los resultados obtenidos.

Tabla 2. Coeficiente de variación de fabricación.

\begin{tabular}{cccccccc}
\hline & $\begin{array}{c}\text { Tiempo de prueba } \\
\text { Emin) }\end{array}$ & $\begin{array}{c}\text { Intervalo de } \\
\text { presiones (bar) }\end{array}$ & $\begin{array}{c}\text { Presión } \\
\text { de prueba } \\
\text { (bar) }\end{array}$ & $\begin{array}{c}\text { Caudal } \\
\text { nominal } \\
(1 / \mathrm{h})\end{array}$ & $\begin{array}{c}\text { Caudal } \\
\text { medio (1/h) }\end{array}$ & $\begin{array}{c}\text { Desviación } \\
\text { Estándar }\end{array}$ & $\begin{array}{c}\text { Coeficiente } \\
\text { Variación }(\%)\end{array}$ \\
\hline Amnondrip 1.1 & 10 & $1,0-4,0$ & 2,50 & 1,10 & 1,07 & 0,030 & 2,84 \\
Amnondrip 1.6 & 10 & $1,0-4,0$ & 2,50 & 1,60 & 1,57 & 0,050 & 3,21 \\
Naan 1.6 & 10 & $0,5-3,0$ & 1,75 & 1,60 & 1,72 & 0,070 & 4,06 \\
Naan 2.2 & 10 & $0,5-3,0$ & 1,75 & 2,20 & 2,45 & 0,079 & 3,22 \\
Clicktif 2.0 & 10 & $0,5-4,0$ & 2,25 & 2,00 & 2,00 & 0,075 & 3,77 \\
Clicktif 8.0 & 4 & $0,5-4,0$ & 2,25 & 8,00 & 7,93 & 0,171 & 2,16 \\
Topdrip 1.0 & 10 & $0,4-2,5$ & 1,45 & 1,00 & 1,16 & 0,040 & 3,44 \\
Corona 4.0 & 5 & $0,5-4,0$ & 2,25 & 4,00 & 4,23 & 0,157 & 3,72 \\
Tifdrip 2.0 & 10 & $0,5-3,0$ & 1,00 & 2,00 & 2,00 & 0,248 & 12,45 \\
Taldrip 1.0 & 10 & $1,0-1,8$ & 1,00 & 1,00 & 1,11 & 0,028 & 2,55 \\
Taldrip 1.7 & 10 & $1,0-1,8$ & 1,00 & 1,70 & 1,74 & 0,044 & 2,53 \\
New GR 2.0 & 10 & $0,5-4,5$ & 1,00 & 2,00 & 2,28 & 0,068 & 2,98
\end{tabular}

La mayoría de los emisores probados estuvieron dentro del límite aceptable según la norma, es decir, menores al $7 \%$. El que presentó un menor valor y en consecuencia una mayor uniformidad en su construcción, fue el modelo autocompensado Clicktif de $8,01 / \mathrm{h}$ con $2,16 \%$. En contrapartida el modelo con un mayor valor fue la línea emisora no compensada modelo Tifdrip 2.0, con $12,45 \%$. Los valores de coeficiente de variación de los emisores restantes fluctuaron entre $2 \%$ y $4 \%$.

Para el modelo Topdrip, que presentó una diferencia significativa entre el caudal hallado y el nominal, se halló un coeficiente de variación de $3,44 \%$, lo cual da un indicativo de las altas descargas que tendrá una muestra de este lote entre emisor y emisor, debido al bajo valor de este coeficiente.

\section{Ecuación de descarga y coeficiente de correlación}

En la Tabla 3 se muestran las características hidráulicas de los emisores evaluados.

Se puede apreciar que 8 de los emisores evaluados presentan coeficientes de determinación $\left(\mathrm{r}^{2}\right)$ altos, lo que indica que la variable independiente (presión) ejerció una alta influencia sobre la variable dependiente (caudal), existiendo por tanto una alta dependencia entre estas variables evaluadas en laboratorio. Lo contrario ocurre con los modelos Naan 2.2, Clicktif 2.0, Clicktif 8.0 y sobre todo con el modelo de manguera Corona 4.0, la cual presentó una relación de 2,38\% entre las variables caudal y presión.

En el caso del exponente de gotero $(x), 7$ de los 12 modelos (todos autocompensados) presentaron una correlación negativa muy cercana a cero entre el caudal y la presión, lo que significa que mientras la presión a la que fue sometida la línea fue en aumento el caudal tendió a la disminución. En los restantes modelos, 4 no compensados y uno compensado (Naan 1.6), la correlación entre estas variables fue positiva, es decir que cuando las presiones aumentaron, los caudales tendieron a aumentar.

Finalmente, en 8 de los 12 modelos de emisores (todos compensados), los coeficientes del gotero $(K)$ tuvieron valores cercanos a los valores del caudal nominal y los 4 emisores restantes (no compensados) presentaron valores inferiores al caudal teórico. 
Tabla 03. Características hidráulicas de los emisores.

\begin{tabular}{ccccc}
\hline $\begin{array}{c}\text { Modelo del } \\
\text { Gotero }\end{array}$ & $K$ & $x$ & $\begin{array}{c}\text { Coeficiente } \\
\text { de } \\
\text { correlación } \\
\left(\mathrm{r}^{2}\right)\end{array}$ & $\begin{array}{c}\text { Cinta/ } \\
\text { Manguera }\end{array}$ \\
\hline Amnondrip 1.1 & 1,1559 & $-0,023$ & 0,8406 & Manguera \\
Amnondrip 1.6 & 1,6266 & $-0,014$ & 0,8840 & Manguera \\
Naan 1.6 & 1,4600 & 0,0462 & 0,9440 & Manguera \\
Naan 2.2 & 2,6259 & $-0,0200$ & 0,5852 & Manguera \\
Clicktif 2.0 & 2,0223 & $-0,0015$ & 0,4516 & Manguera \\
Clicktif 8.0 & 8,0148 & $-0,0125$ & 0,6457 & Manguera \\
Topdrip 1.0 & 1,2249 & $-0,0290$ & 0,9797 & Manguera \\
Corona 4.0 & 4,2483 & $-0,0020$ & 0,0238 & Manguera \\
Tifdrip 2.0 & 1,064 & 0,2740 & 0,9957 & Manguera \\
Taldrip 1.0 & 0,4126 & 0,4306 & 0,9983 & Cinta \\
Taldrip 1.7 & 0,5996 & 0,4481 & 0,9993 & Cinta \\
New GR 2.0 & 0,7565 & 0,4809 & 0,9992 & Manguera \\
\hline
\end{tabular}

\section{Prueba de hipótesis de los goteros evaluados en} laboratorio

Considerando la hipótesis nula: los goteros presentan un valor medio del caudal igual a lo publicado en los catálogos de los fabricantes y asumiendo en todos los casos la desviación estándar muestral como poblacional, a continuación se presentan las pruebas de hipótesis.

\section{Manguera integrada modelo Amnondrip 1.1}

Ho: $\mathrm{q}=1,1 \mathrm{l} / \mathrm{h}$, para $n=30$

El intervalo de confianza es $1,09<\mu<1,11$. El puntaje promedio poblacional se encuentra entre estos valores, con un nivel de confianza al $95 \%$. Como el caudal medio obtenido de la muestra fue de $1,07 \mathrm{l} / \mathrm{h}$, se rechaza la hipótesis nula, por lo tanto los goteros no presentan un valor medio del caudal igual a lo publicado en los catálogos de los fabricantes.

\section{Manguera integrada modelo Amnondrip 1.6}

Ho: $\mathrm{q}=1,61 / \mathrm{h}$, para $n=30$

El intervalo de confianza es $1,58<\mu<1,62$. El puntaje promedio poblacional se encuentra entre estos valores, con un nivel de confianza al 95\%. Como el caudal medio obtenido de la muestra fue de $1,57 \mathrm{l} / \mathrm{h}$, se rechaza la hipótesis nula, es decir, los goteros no presentan un valor medio del caudal igual a lo publicado en los catálogos de los fabricantes.

\section{Manguera integrada modelo Naan 1.6}

Ho: $\mathrm{q}=1,6 \mathrm{l} / \mathrm{h}$, para $n=30$

El intervalo de confianza es $1,57<\mu<1,63$. El puntaje promedio poblacional se encuentra entre estos valores, con un nivel de confianza al $95 \%$. Como el caudal medio obtenido de la muestra fue de $1,72 \mathrm{l} / \mathrm{h}$, se rechaza la hipótesis nula, por lo tanto los goteros no presentan un valor medio del caudal igual a lo publicado en los catálogos de los fabricantes.

\section{Manguera integrada modelo Naan 2.2}

Ho: $\mathrm{q}=2,2 \mathrm{l} / \mathrm{h}$, para $n=30$

El intervalo de confianza es $2,17<\mu<2,23$. El puntaje promedio poblacional se encuentra entre estos valores, con un nivel de confianza al 95\%. Como el caudal medio obtenido de la muestra fue de $2,45 \mathrm{l} / \mathrm{h}$, se rechaza la hipótesis nula, es decir, los goteros no presentan un valor medio del caudal igual a lo publicado en los catálogos de los fabricantes.

\section{Gotero insertado modelo Clicktif 2.0}

Ho: $\mathrm{q}=2,0 \mathrm{l} / \mathrm{h}$, para $n=30$

El intervalo de confianza es $1,97<\mu<2,03$. El puntaje promedio poblacional se encuentra entre estos valores, con un nivel de confianza al 95\%. Como el caudal medio obtenido de la muestra fue de $2 \mathrm{l} / \mathrm{h}$, se acepta la hipótesis nula, es decir, los goteros presentan un valor medio del caudal igual a lo publicado en los catálogos de los fabricantes.

\section{Gotero insertado modelo Clicktif 8.0}

Ho: $\mathrm{q}=8,0 \mathrm{l} / \mathrm{h}$, para $n=30$

El intervalo de confianza es 7,94 $<\mu<8,06$. El puntaje promedio poblacional se encuentra entre estos valores, con un nivel de confianza al $95 \%$. Como el caudal medio obtenido de la muestra fue de $7,93 \mathrm{l} / \mathrm{h}$, se rechaza la hipótesis nula, por lo tanto, los goteros no presentan un valor medio del caudal igual a lo publicado en los catálogos de los fabricantes.

\section{Manguera integrada modelo Topdrip 1.0}

Ho: $\mathrm{q}=1,0 \mathrm{l} / \mathrm{h}$, para $n=30$

El intervalo de confianza es $0,98<\mu<1,02$. El puntaje promedio poblacional se encuentra entre estos valores, con un nivel de confianza al 95\%. Como el caudal medio obtenido de la muestra fue de $1,16 \mathrm{l} / \mathrm{h}$, se rechaza la hipótesis nula, es decir, los goteros no presentan un valor medio del caudal igual a lo publicado en los catálogos de los fabricantes.

\section{Gotero insertado modelo Corona 4.0}

Ho: $\mathrm{q}=4,0 \mathrm{l} / \mathrm{h}$, para $n=30$

El intervalo de confianza es 3,94 $<\mu<4,06$. El puntaje promedio poblacional se encuentra entre estos valores, con un nivel de confianza al $95 \%$. Como el caudal medio obtenido de la muestra fue de $4,23 \mathrm{l} / \mathrm{h}$, se rechaza la hipótesis nula, por lo tanto, los goteros no presentan un valor medio del caudal igual a lo publicado en los catálogos de los fabricantes. 


\section{Manguera integrada modelo Tifdrip 2.0}

Ho: $\mathrm{q}=2,0 \mathrm{l} / \mathrm{h}$, para $n=30$

El intervalo de confianza es $1,92<\mu<2,09$. El puntaje promedio poblacional se encuentra entre estos valores, con un nivel de confianza al 95\%. Como el caudal medio obtenido de la muestra fue de $2,0 \mathrm{l} / \mathrm{h}$, se acepta la hipótesis nula, es decir, los goteros presentan un valor medio del caudal igual a lo publicado en los catálogos de los fabricantes.

\section{Cinta integrada modelo Taldrip $\mathbf{1 . 0}$}

Ho: $\mathrm{q}=1,0 \mathrm{l} / \mathrm{h}$, para $n=30$

El intervalo de confianza es $0,99<\mu<1,01$. El puntaje promedio poblacional se encuentra entre estos valores, con un nivel de confianza al 95\%. Como el caudal medio obtenido de la muestra fue de $1,11 \mathrm{l} / \mathrm{h}$, se rechaza la hipótesis nula, por lo tanto, los goteros no presentan un valor medio del caudal igual a lo publicado en los catálogos de los fabricantes.

\section{Cinta integrada modelo Taldrip 1.7}

Ho: $\mathrm{q}=1,7 \mathrm{l} / \mathrm{h}$, para $n=30$

El intervalo de confianza es $1,68<\mu<1,72$. Es decir, el puntaje promedio poblacional se encuentra entre estos valores, con un nivel de confianza al 95\%. Como el caudal medio obtenido de la muestra fue de $1,74 \mathrm{l} / \mathrm{h}$, se rechaza la hipótesis nula, es decir, los goteros no presentan un valor medio del caudal igual a lo publicado en los catálogos de los fabricantes.

\section{Manguera integra modelo New GR 2.0}

Ho: $\mathrm{q}=2,0 \mathrm{l} / \mathrm{h}$, para $n=25$

Como la muestra es menor a 30 se realiza la prueba con una distribución $\mathrm{t}$ de Student, con un nivel de confianza del 95\%, siendo el valor de 2,045.

El intervalo de confianza es 1,97< $<<2,03$. El puntaje promedio poblacional se encuentra entre estos valores, con un nivel de confianza al $95 \%$. Como el caudal medio obtenido de la muestra fue de 2,28 1/h, se rechaza la hipótesis nula, por lo tanto, los goteros no presentan un valor medio del caudal igual a lo publicado en los catálogos de los fabricantes.

\section{Conclusiones}

Los emisores evaluados presentaron un flujo turbulento y autocompensado. La mayoría de los emisores probados estuvieron dentro del límite aceptable del $C V$ según la norma ISO 9261 (menores al 7\%). El modelo que superó este valor límite fue la línea emisora no compensada modelo Tifdrip ( $q n=2,0$ l/h), el cual presentó un valor de $12,45 \%$, que supera a los demás emisores compensados evaluados y una diferencia significativa entre la curva teórica y práctica. Los signos del exponente del gotero de los modelos evaluados indican la relación de proporcionalidad entre las dos variables intervinientes: caudal y presión. En 5 de los 12 modelos se obtuvo un coeficiente con signo positivo, lo cual indica una relación directa entre el caudal y presión en sus respectivas curvas características, y viceversa en los restantes modelos de goteros. Según las evaluaciones estadísticas, para un nivel de confianza del $95 \%$, efectuadas para cada uno de los emisores, solamente los emisores Clicktif 2.0 y Tifdrip 2.0 presentan un valor medio del caudal igual a lo publicado en los catálogos de los fabricantes; en los 10 modelos restantes, el valor medio del caudal de los goteros no es igual a lo publicado en los catálogos de los fabricantes.

\section{Literatura citada}

Fuentes, Y. J. L. 2003. Técnicas de riego. Ministerio de Agricultura, Pesca y Alimentación. Ediciones MundiPrensa. Madrid. 483 p.

ISO 9261.2004. Equipo de irrigación agrícola - Emisores y tubo de emisión - Especificación y métodos de prueba.

Keller, J. y Bliesner, R. 1990. Sprinkle and trickle irrigation. Ediciones Van Nostrand Reinhold. New York, Estados Unidos. $652 \mathrm{p}$.

Medina San Juan, J. A. 1997. Riego por goteo. Ediciones Mundi-Prensa. Madrid, España. 302 p.

Merino, T. 2007. Intervalos de confianza. Santiago, Chile. Consultado en http://escuela. med.puc.cl/recursos/ recepidem/EPIANAL9.HTM.

Pizarro, F. 1996. Riego localizado de alta frecuencia (RLAF) goteo, micro aspersión, exudación. Ediciones Mundi-Prensa. Madrid. España. 510 p. 